\title{
Grouping Social Media Users Through Goffman's Dramaturgical Approach and the Preservation of Self Presentation
}

\author{
Yaqun Qian ${ }^{1, *}$ \\ ${ }^{I}$ German Culture and Management, Royal Holloway, University of London, Surrey, UK \\ ${ }^{*}$ Corresponding author. Email: guanghua.ren@gecacademy.cn
}

\begin{abstract}
The transformation of social media has identified the modern self in the age of New media. With the accumulation of users' numbers and merged functions of social media platforms, it is worthy to be re-examined that whether traditional semiotics and sociological explanations are still valid for the new self-presentation behavior. To understand how users in the new media age gain their self-identities through social media platforms, the account posted content analysis and user interactions are put into scrutiny by this study. The Chinese social media application Wechat is available for study purposes due to its large user number and its users' alternative options to choose other social applications such as Instagram. By applying Goffman's dramaturgical theory onto the sociological observation on users' behaviors of Chinese successful social platform and communicational applications Wechat, this study argues that the self-presentation of the new media users is still coherent to the stage-persona analysis from Goffman. It is observed that the selectiveness and the consciousness of users' performing self have been changed by the environment of social media innovations. The unique case of the quasi- Instagram social content Finsta is also introduced in this study.
\end{abstract}

Keywords: Self-presentation, social media, Goffman 's dramaturgical theory

\section{INTRODUCTION}

Accurate self-representation is essential to constructing one's identity and desired image for selected groups to view. Goffman's dramaturgical approach includes 'encounters' and 'performers' that suggest multi-dimensions within an individual's presentation exist for a targeted group of audience. The theoretical metaphor of front-stage and back-stage is Goffman's prominent approach of shifting words and abstractions between settings leading to Goffman's central idea of one's encounter performance [1]. The sole purpose of particular sides from an individual's behavior is only accessible by a chosen group of viewers further demonstrates the grants and limits an individual manipulates to perfect their performance. Two central influences being surrounding observers and the environment play a crucial role in shaping the actor's performance. The performances will differ depending on the environment, such as an actor's behavior backstage will be not identical to the front stage behavior [2].
Extending from Goffman's dramaturgical approach, the correlation between an individual's decision to perform a certain act and the corresponding stage's audience deeply controls the actor's judgment. It is apparent that the audience groups match up with the types of the stage that the actor is performing on, however, it is not indicated that the audience is in a fixed position. Given that the actor can travel from front to backstage, the question of if the audience can rotate or switch freely from their original groups to access a different performance is raised. With the emergence of popular social media usage, Goffman's notions of performance are merely moved online with the same groups of interaction and roles. The movement of audience grouping remains to be unclear in a performance occurring on and offline raises concerns regarding if the audience grouping follows each individual's rigid rules.

This paper aims to investigate the flow of audiences in Goffman's dramaturgical approach and explore possible triggers that permit the shift. The interconnection between an actor's performance and the 
decision to add or remove from audience groups, the findings will serve to provide a holistic outlook for the individual to understand and reflect upon oneself, additionally make flexible adjustments to maximize the performance effect.

\section{GOFFMAN'S THEORY AND THE CONCEPT OF STAGE}

Goffman's notion of dramaturgy compares real life to theater stages where individuals wear various role reflecting masks. Thus, the essential factor in Goffman's dramaturgy comparison is the actor's role in which refers to a specific image an individual desire to express [3]. Goffman proclaimed that in-person interaction "is never merely and not always a form of communication" [4]. The reference of face-to-face interaction is employed to indicate Goffman's focus on people's daily behavior but on a micro-scale which the individuals are not aware of, such as their manner of speech in greetings or the physical contact [5]. It is also applicable to observe the details and settings online to examine how one distinguishes their audience groups. Goffman's dramaturgical model assumes that functioning roles such as the actor and the audience must be present and enabled before a performance, whether in a focused or unfocused encounter, both roles assist each other to an extent to enforce the performance. The fronts circle the audience's presence and push the individual to adapt based on the desired effect on the grouping. Goffman does not restrict the front or backstage to a set location or point, therefore the stages have unbounded possible placements and any site could be set up as a stage before a performance [6].

The ubiquitousness of social media in modern society amongst the younger population has contributed to a change of self-identity and image construction, thus leading to the preservation of oneself's presentation. Obtaining profiles and data from the one eastern and one western social media applications being Wechat and Instagram respectively, the visibility of posts and accessibility of users will be compared and analyzed to elucidate how the selection of audience and grouping facilitate a performance. The online behavior and content will also be included when interpreting the suitability for an individual's designated audience groups. Followed by a questionnaire with active users of the two applications to acquire the personal reasoning and process of audience grouping.

WeChat is a prominent Chinese social application that has over 1.2 billion active users. The wide coverage of WeChat includes not only conversational functions but also incorporates a feed named 'Moments' where one can upload photos or text. The combination of chatting plus social media accelerated WeChat's popularity and usage across the nation. In 2017, a new function under privacy was introduced to limit the visibly of a user's posts to the 'Last Three Days 'and sparked immediate disputes on reasons and hidden meanings when an individual choose to do so [7]. One possible explanation to which up till 2021, more than 200 million users have chosen the three-day moment's visibility is the increased awareness of online identity theft or fraud. Countless articles are complaining that some viewers are offended when they realize they have limited access to a user's moments and often use the words "trust" and "friendship" to argue for further accessibility. Users have responded that it is a protective function that not only shields privacy from internet strangers but also refrains from appearing to be a different presentation than the one previously built for certain viewers.

\section{THE NEW FUNCTION}

With WeChat's trend of limiting viewer accessibility, a similar trend of Gen Z's creating a 'fake' profile named 'Finsta' which is a shortening of 'FakeInsta' emerged on Instagram. This is more direct audience segregation compared to WeChat's functions and a Finsta is a second Instagram account that reveals personal matters on a usually private account that only accepts a small number of people. As BBC interviewed a Finsta account user Jamie, he expressed his reasoning like the following, "On my Finsta, the content ranges from embarrassing photos that I wouldn't dream of sharing on my main account... My main Instagram is used to present myself in a certain way... I'm able to be my silly self with my friends, while still maintaining my image on the other [main] account." From Jamie's statement, the main Instagram falls under a front stage and focused encounter where Jamie is aware and aims to preserve his built image to the public. However, the lack of backstage on Instagram lead Jamie and many others to create a second secretive and selective profile to post more casual matters that are only viewable for the approved crowd. It does happen when a member from the public attempts to request permission to access the Finsta account which gives the audience a portion of power to request additional access. Sherwood reports that from the interviewed Finsta users Jamie and Jolyon, Finsta serves as escapism and a medium to share content with selected friends [8].

The resemblance of Goffman's fronts and audience is strongly present with both WeChat and Instagram's trend of limiting performance fronts. These popular social media trends of restricting the accessibility of content and limiting audience entries demonstrate the phenomenon of the mass population is aware of their unfocused encounters that could pose a threat by accidentally revealing a front. From the gathered results and online profile analysis, the manipulation of viewer accessibility about Goffman's dramaturgical model may 
imply a potential circulation of regular audience groups in an individual's performance.

Goffman emphasizes that conflicts may arise when fronts clash with one another, flipping the situation and conjecturing the audience groups being disarranged would undoubtedly cause a significant impact. Beginning with how the initial groupings of an individual's audience are appointed, the addition or removal or jump of one or a few members of the audience from one group to another provides a trail that can be followed for evaluation of the ultimate influence upon one's fronts. The change in audience grouping may occur without acknowledgment, identical to unfocused encounters, an individual can be unaware of the ejection of certain members. The consequences of audience rearrangement remain unexplored and the issues regarding accidental or deliberate exposure of fronts to unapproved viewer groups in Goffman's dramaturgical model. Does the front-stage viewer get permanently removed from the audience group or an accidental performance or front reveal is simply neglected to ensure coherence and fairness of other members? A common case observed includes, an individual having an uncontrolled sudden emotional scene in an office setting with their work associates witnessing the event. The majority of working adults would group colleagues or office superiors further down the line indicating the unwillingness to form a deeper relationship or reveal personal matters in front of them. The unwillingness began simultaneously as the job, the individual builds a professional front that is unavailable to the audience group of family members and vice versa. From visuals and media such as films, it is portrayed as difficult to permit another group member to witness a different performance. The intended audience group may not always be reached and in this case, the actor can switch fronts or regroup the audience to ensure the performance continues.

Previous research has proven that individuals view their online identity equivalently with their real-life character, the only variance is the context that oneself is in. Further investigation concurs by the indication that the studies subjects expressed the inclination to keep their online character in close proximity of their real-life persona and eliminate the option of adopting a rand new persona [2]. With the wish to maintain the close relationship between the two identities of oneself, the goal of grouping the audience in a similar method can be deduced. Such as grouping identical characteristics or according to the maintenance of one's established image required.

To portray a precise self-representation upon the right groups of audience requires not only constant vigilance but only flexibility to reposition certain groups. From Goffman's dramaturgical approach, it is observed to contain the direct way being focused encounter often on a frontal stage serving as the unrestricted facade. It is the latter of unfocused encounters occurring backstage for initially selected audience groups and the regrouping that demands further investigation to help gain a deeper understanding of the purpose of individuals shifting fronts and fundamentally improving human relationships. Adopting Goffman's terminology, life will be an ongoing series of performances and with more knowledge regarding the available fronts and circulation or limitation of audience grouping, both the actors and audience will be able to assist each other to secure a safe and reciprocated performance whether on or offline.

\section{DISCUSSIONS}

Users on the social media platforms Instagram and WeChat have been observed to limit accessibility and progressively selective of the targeted audience. Which could be interpreted as means of seeking more control over their self-representation and being concerned with the audience's reaction. Not only do the users have identical traits, but both platforms also share the great similarity in terms of designing and offering homogeneous functions. The primary drive for Instagram and WeChat users inclining towards a more private and cautious approach to their online representation stems from a closely correlated origin. The prevalence of social media and the internet increased the general public's consciousness of their online identity thus, leading the users to a rather conservative decision today and the apps to provide those functions to cater to the new emotional demands of their customers.

"Three days visible" and "Selected contacts visible" in WeChat have gained popularity throughout user groups excluding the age factor in the past years [9]. The two functions are essentially alike, both offering to include and exclude audience groups of choice. Interviewees from a study reported that the two functions have assisted them significantly to manipulate their ideal self-presentation as time passes. Users are persistent with having control over their content visibility which could be beneficial for users in diverse networks [10].

Despite the users on Instagram and WeChat being usually amongst the younger population, the difference is that WeChat "Three days visible" is used by all age groups from senior citizens to adolescents whereas "Finsta" is created by the younger population. Finsta user-group consists of main teenagers as a survey revealed that the central motive behind the creation of Finsta is to prevent context collapse. Similar to WeChat users' ability to control their content visibility, when the functions are not applied it is possible for context to collapse and bring disorder when the incorrect audience views a different performance and behavior. It is also 
discerned that Finsta allows users to act freely and less restricted that resulting in unrestrained self-expression and presentation [11]. Therefore, it is able to generate less worrying sensations regarding the presence of certain members in an audience and instead provides a safe space that ensures user each member in the audience is handpicked. It is evident that WeChat and Instagram both permit users to carefully select one member each time to include or exclude which dissects Goffman's audience concept. By handpicking the new audience, users scrutinize and are able to demonstrate a deeper layer of themselves.

Both user groups have demonstrated increasing selectiveness for their audience and consciousness to use different accounts to symbolize which stage they are performing on. From the user's age groups and in relation to the socio-emotional selectivity theory, it proves that both user groups rearranged their priority to relate to a certain significance as a conducted online survey discovered that the received responses from an individual's online network heavily influences their well-being [12]. This trend, if Goffman's theory will be continuingly coherent with the social media technological transformations, is not limited to generation $\mathrm{Z}$ or some specific groups. The performing self in the new media age does have its stable pattern.

\section{CONCLUSION}

The diversity of self-presentation is always understood as a modern civilization's success while the innovation of communicational technology is of no doubt another one. With Goffman's dramaturgical theory applied to the communicational behaviors of social media platform Wechat users, this article depicts the changing picture of how modern social habits and customs are transformed by innovative modern technology. With more and more privacy exposure and better chances for oneself to be seen or heard, the performing self, or the self-identification process has shown an opposite way by the increasing selectiveness for audience and consciousness to use different accounts to symbolize the stage. There is one hidden assumption under this changing picture that the social environment, the socialized self, and modern media techniques are not passing through the same path into the new media age modernity. The awareness of self visibility is perhaps a tricky concept when concerning new media social habits and the new media economic success. Goffman's dramaturgical description fits the self-presentation in the new media platform user performance, however, the self and user groups may divide from within soon. The multi-universe structure and even meta-universe will continue the technological strike on social media transformation and the performing self on its quasimodern stage. The seemingly stable function of self- presentation and the individual control of self visibility and audience selectiveness will meet more challenges.

\section{REFERENCES}

[1] Mueller, F., 2018. Taking Goffman seriously: Developing Strategy-as-Practice. Critical Perspectives on Accounting, 53, pp.16-30.

[2] Bullingham, L. and Vasconcelos, A., 2013. 'The presentation of self in the online world': Goffman and the study of online identities. Journal of Information Science, 39(1), pp.101-112.

[3] Kivisto, P., 2012. Illuminating Social Life: Classical and Contemporary Theory Revisited. 6th ed. SAGE Publications, pp.300, 367.

[4] Goffman, E., 1986. Strategic interaction. Philadelphia: University of Pennsylvania Press, pp.6,7.

[5] EPSTEIN-CORBIN, SEAN. “'THE SENTIMENT OF RATIONALITY': WILLIAM JAMES AND THE SENTIMENTAL TRADITION." William James Studies 13, no. 1 (2017): 27-48. http://www.jstor.org/stable/26203814.

[6] Hogan, B., 2010. The Presentation of Self in the Age of Social Media: Distinguishing Performances and Exhibitions Online. Bulletin of Science, Technology \& Society, 30(6), pp.377-386.

[7] Sohu.com. 2021. "From WeChat's [Three day visible' function, analysis of the hidden reason behind it" [online] Available at: <https://www.sohu.com/a/156224971_114819>

[Accessed 26 September 2021].

[8] Sherwood, J., 2019. Why Generation Z are choosing 'Finsta' over 'Insta'. [online] BBC News. Available at: <https://www.bbc.com/news/blogs-trending49852428> [Accessed 27 September 2019].

[9] Zheng, G. and Zhao, Z., 2020. Social media users' behavior of setting limits in information sharing: A heuristic study based on WeChat moments. Proceedings of the Association for Information Science and Technology, 57(1).

[10] Huang, X., Vitak, J. and Tausczik, Y., 2020. "You Don't Have To Know My Past": How WeChat Moments Users Manage Their Evolving SelfPresentation. Proceedings of the 2020 CHI Conference on Human Factors in Computing Systems.

[11] Dewar, S., Islam, S., Resor, E. and Salehi, N., 2019. Finsta. Extended Abstracts of the 2019 CHI Conference on Human Factors in Computing Systems. 
[12] Rui, J., Cui, X., Xu, Q. and Yu, N., 2021. How public interactions via WeChat moments predict the emotional well-being of Chinese seniors and emerging seniors: The moderating roles of perceived self-network discrepancy and age. Cyberpsychology: Journal of Psychosocial Research on Cyberspace, 15(3). 